\title{
Matrix compactification on orientifolds
}

\author{
Pei-Ming $\mathrm{Ho}^{*}$ \\ Department of Physics, National Taiwan University, Taipei 10764, Taiwan \\ Yong-Shi $\mathrm{Wu}^{\dagger}$ \\ Department of Physics, University of Utah, Salt Lake City, Utah 84112
}

(Received 13 January 1999; published 14 June 1999)

\begin{abstract}
Generalizing previous results for orbifolds, in this paper we describe the compactification of the matrix model on an orientifold which is a quotient space $\mathbf{R}^{d} / \Gamma$ as a Yang-Mills theory residing on a quantum space. The information of the compactification is encoded in the action of the discrete symmetry group $\Gamma$ on Euclidean space $\mathbf{R}^{d}$ and a projective representation $U$ of $\Gamma$. The choice of Hilbert space on which the algebra of $U$ is realized as an operator algebra corresponds to the choice of a physical background for the compactification. All these data are summarized in the spectral triple of the quantum space. [S0556-2821(99)06512-1]
\end{abstract}

PACS number(s): 11.25.Mj, 11.25.Sq

\section{INTRODUCTION}

The original matrix model [1] was formulated as a microscopic model for $\mathrm{M}$ theory in 11-dimensional spacetime. To describe the real world one needs to understand how to compactify the theory to lower dimensions. In the absence of a rank-3 antisymmetric tensor field background, the description of the compactification of the matrix model is similar to that of D-branes in compactified string theory [2-5]. However, if such a background is present, we have to use the concepts of noncommutative geometry to describe the compatified matrix model, as first shown by Connes, Douglas, and Schwarz [6] for toroidal compactification.

In Refs. [7,8] we generalized the noncommutative geometric description of D-branes in Ref. [9] to incorporate orbifold compactifictions, including the possibility for discrete torsion. In particular, in Ref. [8] we gave a general formulation for interpreting the matrix model compactified on manifolds and orbifolds as gauge field theories on quantum spaces. In this paper we proceed to describe the compactified matrix model on an orientifold, which is the quotient of the flat infinite space by the action of a discrete symmetry group $\Gamma$ of the matrix model, which contains the operation of matrix transposition. More precisely, we will allow a $\mathbf{Z}_{2}$ grading of $\Gamma$, which corresponds to transposition of the matrix variables $X^{\mu}$ and $\Psi^{\alpha}$. If the matrix model action is interpreted as the low energy effective action of D-branes, the transposition represents the worldsheet parity transformation of the open strings connecting the D-branes. In this way, the quotient space is an orientifold if $\Gamma$ has such nontrivial $\mathbf{Z}_{2}$ grading.

Our presentation will be such that the quotient space can be a manifold orbifold, or orientifold. This paper also contains some technical details which were not included in our previous short paper [8]. We review how the quotient conditions arise from taking quotient of the flat space in Sec. II. We will see that the quotient conditions need to be supplemented by the information of the $U$ algebra (20) in order to

\footnotetext{
*Email address: pmho@phys.ntu.edu.tw

${ }^{\dagger}$ Email address: wu@mail.physics.utah.edu
}

completely specify the compactification. In Sec. III we show that the moduli space of the $U$ algebra is a $\mathbf{Z}_{2}$-graded second Hochschild cohomology group $H^{2}(\Gamma, U(1))$. For the trivial bundle on the dual quantum space, the most general solutions to the quotient conditions are obtained in Sec. IV. Following a brief review of noncommutative geometry in Sec. $\mathrm{V}$, we give a noncommutative geometric description of the dual quantum space in Sec. VI. Then we examine residual symmetries in the compactified matrix model in Sec. VII, and give a few examples to demonstrate how the formulation actually works in Sec. VIII. Finally in Sec. IX we comment on several possible generalizations of our formulation of matrix model compactifications.

\section{QUOTIENT CONDITIONS}

Consider the compactification of the matrix model on a space which is the quotient of the flat space $\mathbf{R}^{d}$ over a discrete group $\Gamma$. Taking the quotient makes sense for the matrix model only if $\Gamma$ is a symmetry of the matrix model. Let the matrix variables be $\left(X^{\mu}, \Psi^{\alpha}\right)$, where $\mu=0,1, \ldots, 9$ and $\alpha=1,2, \ldots, 16$ are the Lorentz indices for spin 1 and spin $1 / 2$ representations, respectively, in ten-dimensional Minkowski space in the light-cone formulation for $\mathrm{M}$ theory. In addition to the usual isometry group $G_{0}$ of the superspace, which is the super-Poincaré group times the spacetime reflections and reversals, the matrix transposition of these variables is also a symmetry of the matrix model. In the D0brane interpretation for the original matrix model [1], the matrix index labels different D0-branes, so that off-diagonal elements of $X^{\mu}$ and $\Psi^{\alpha}$ describe open strings stretched between the D0-branes. Therefore taking transposition means string world sheet parity transformation. Since $X^{\mu}$ and $\Psi^{\alpha}$ are Hermitian matrices, taking matrix transposition is equivalent to taking complex conjugation:

$$
X^{\mu} \rightarrow X^{\mu *}, \quad \Psi^{\alpha} \rightarrow \Psi^{\alpha *},
$$

where * denotes complex conjugation. In the following, we will use this to represent string world sheet parity transformation. 
Thus we take $\Gamma$ naturally to be a subgroup of $G_{0} \times \mathbf{Z}_{2}$, and any $g \in \Gamma$ can be written as $\left(g_{0}, c(g)\right)$, where $g_{0} \in G_{0}$ and $c(g) \in \mathbf{Z}_{2}$. The space we take as quotient is thus actually the superspace times a factor of $\mathbf{Z}_{2}$ (or two copies of superspace), on which the world sheet parity acts. The $Z_{2}$ structure has to be compatible with the multiplication in $G_{0}$ :

$$
c\left(g_{1} g_{2}\right) \equiv c\left(g_{1}\right)+c\left(g_{2}\right)(\bmod 2),
$$

where we used the representation with $c(g)=0,1$. The quotient space $\mathbf{R}^{d} / \Gamma$ is an orientifold if $c(g)=1$ for some $g$ $\in \Gamma$.

The action of $g$ is

$$
\begin{aligned}
& X^{\mu} \rightarrow \Phi_{g}^{\mu}(X), \quad \Psi^{\alpha} \rightarrow \Phi_{g}^{\alpha}(\Psi) \text { if } c(g)=0, \\
& X^{\mu} \rightarrow \Phi_{g}^{\mu}\left(X^{*}\right), \quad \Psi^{\alpha} \rightarrow \Phi_{g}^{\alpha}\left(\Psi^{*}\right) \text { if } c(g)=1 .
\end{aligned}
$$

In the above $\Phi_{g}$ is of the form

$$
\Phi_{g}^{\mu}(X)=R(g)_{\nu}^{\mu} X^{\nu}+a(g)^{\mu}, \Phi_{g}^{\alpha}(\Psi)=\Lambda(g)_{\beta}^{\alpha} \Psi^{\beta},
$$

where $(R(g), a(g))$ and $\Lambda(g)$ are representations of $G_{0}$. The map $\Phi_{g}$ does not depend on $c(g)$ but only on $g_{0}$.

Two consecutive transformations by $g_{1}$ and $g_{2}$ give

$$
X^{\mu} \rightarrow \Phi_{g_{1}}^{\mu}(X) \rightarrow \Phi_{g_{1}}^{\mu}\left(\Phi_{g_{2}}(X)\right),
$$

which should be equivalent to a single transformation by $g_{1} g_{2}$ :

$$
X^{\mu} \rightarrow \Phi_{g_{1} g_{2}}^{\mu}(X)
$$

It follows that

$$
\begin{aligned}
& R\left(g_{1} g_{2}\right)_{\nu}^{\mu}=R\left(g_{1}\right)_{\sigma}^{\mu} R\left(g_{2}\right)_{\nu}^{\sigma}, \\
& a\left(g_{1} g_{2}\right)^{\mu}=R\left(g_{1}\right)_{\nu}^{\mu} a\left(g_{2}\right)^{\nu}+a\left(g_{1}\right)^{\mu} .
\end{aligned}
$$

Denote the operator of complex conjugation by $\mathcal{C}$ so that

$$
\mathcal{C}^{2}=1, \text { and } \mathcal{C} c=c^{*} \mathcal{C}
$$

for any complex number $c$. Equations (3) and (4) can now be summerized as

$$
X^{\mu} \rightarrow \mathcal{C}^{c(g)} \Phi_{g}^{\mu}(X) \mathcal{C}^{c(g)}, \quad \Psi^{\alpha} \rightarrow \mathcal{C}^{c(g)} \Phi_{g}^{\alpha}(\Psi) \mathcal{C}^{c(g)},
$$

where $\mathcal{C}^{0}=1$ and $\mathcal{C}^{1}=\mathcal{C}$.

Taking the quotient over $\Gamma$ means that we impose an equivalence relation between $\left(X^{\mu}, \Psi^{\alpha}\right)$ and their transformed images (11). Using this equivalence relation, we can divide the superspace into (possibly infinitely) many fundamental regions. Let there be $n$ partons in each fundamental region, and let each fundamental region be labeled by an element in the group $\Gamma$. We can first choose an arbitrary fundamental region and label it by $e$, the unit element in $\Gamma$. Then the group element $g$ transforms the region labeled by $e$ to another region labeled by $g$. Hence the matrix variables acquire extra indices as

$$
X_{\left(g_{1}, i\right)\left(g_{2}, j\right)}^{\mu}, \quad \Psi_{\left(g_{1}, i\right)\left(g_{2}, j\right)}^{\alpha},
$$

where $i, j=1,2, \ldots, n$ are the indices used to label the $n$ partons in each fundamental region, and $g_{1}, g_{2} \in \Gamma$ are the indices used to label the fundamental regions. In the flat covering space from which we take the quotient there are a total of $|\Gamma| \times n$ partons. ${ }^{1}$ This implies that additional degrees of freedom appear upon compactification.

With the notation that $*^{0}$ means the identity operation and *1 means complex conjugation, the equivalence relations are

$$
\begin{aligned}
& X_{\left(g g_{1}, i\right)\left(g g_{2}, j\right)}^{\mu}=R(g)_{\nu}^{\mu} X_{\left(g_{1}, i\right)\left(g_{2}, j\right)}^{\nu *^{c(g)}}+a(g)^{\mu} \delta_{g_{1} g_{2}} \delta_{i j}, \\
& \Psi_{\left(g g_{1}, i\right)\left(g g_{2}, j\right)}^{\alpha}=\Lambda(g)_{\beta}^{\alpha} \Psi_{\left(g_{1}, i\right)\left(g_{2}, j\right)}^{\beta *^{c(g)}} .
\end{aligned}
$$

Note that in D0-brane language the diagonal elements in the matrix variables are interpreted as the coordinates of the $n$ D0-branes, and the off-diagonal elements represent open strings stretching between different D0-branes. Thus if a translation is involved for a given $g$, only the diagonal part of $X^{\mu}$ is shifted by the translation. This is why the factor $\delta_{g_{1} g_{2}} \delta_{i j}$ appears in the last term of Eq. (13).

We can rewrite the matrix equations (13) and (14) simply as

$$
\begin{gathered}
U(g)^{\dagger} X^{\mu} U(g)=\mathcal{C}^{c(g)} \Phi_{g}^{\mu}(X) \mathcal{C}^{c(g)}, \\
U(g)^{\dagger} \Psi^{\alpha} U(g)=\mathcal{C}^{c(g)} \Phi_{g}^{\alpha}(\Psi) \mathcal{C}^{c(g)}
\end{gathered}
$$

by using the matrices ${ }^{2}$

$$
U(g)_{g_{1} g_{2}}=\delta_{g_{1}, g g_{2}},
$$

which is nothing but a regular representation of $\Gamma$.

Define

$$
\mathcal{U}(g)=U(g) \mathcal{C}^{c(g)} ;
$$

then, we have simpler expressions for Eq. (15), (16) as

$$
\mathcal{U}(g)^{\dagger} X^{\mu} \mathcal{U}(g)=\Phi_{g}^{\mu}(X), \mathcal{U}(g)^{\dagger} \Psi^{\alpha} \mathcal{U}(g)=\Phi_{g}^{\alpha}(\Psi) .
$$

In fact, Eqs. (15), (16) can be understood directly as a set of algebraic relations without going through the manipulation of indices in the above arguments. Since the matrix model is a gauge theory, Eqs. (15), (16) mean that the equivalence relations are imposed as gauge transformations. We call Eqs. (15) and (16) or, equivalently, Eq. (19), the quotient conditions. It is important that we should treat the quotient conditions as algebraic relations. We are no longer restricted to define $U(g)$ by Eq. (17), which only serves an illustrative purpose.

\footnotetext{
${ }^{1} \mathrm{By}|\Gamma|$ we mean the number of elements in $\Gamma$.

${ }^{2}$ One can modify Eq. (17) by a phase depending on $g$ and $g_{1}$, but it would be equivalent to Eq. (17) by a unitary transformation.
} 


\section{GROUP COHOMOLOGY}

Without imposing Eq. (17), the compactification is not fully specified by the quotient conditions, although the geometric information is. What we lack is the algebra of $\mathcal{U}$. From Eq. (19) we see that two successive transformations of $X$ and $\Psi$ by $\mathcal{U}\left(g_{1}\right)$ and $\mathcal{U}\left(g_{2}\right)$ are equivalent to a single transformation by $\mathcal{U}\left(g_{1} g_{2}\right)$. If we treat Eq. (19) as the only constraint on $X$ and $\Psi,\{\mathcal{U}(g)\}$ has to be a projective representation of $\Gamma[10,2]$ :

$$
\mathcal{U}\left(g_{1}\right) \mathcal{U}\left(g_{2}\right)=\mathcal{U}\left(g_{1} g_{2}\right) e^{i \alpha\left(g_{1}, g_{2}\right)} .
$$

In terms of $U(g)$ it is

$$
U\left(g_{1}\right) U\left(g_{2}\right)^{*\left(g_{1}\right)}=U\left(g_{1} g_{2}\right) e^{i(-1)^{c\left(g_{1} g_{2}\right)} \alpha\left(g_{1}, g_{2}\right)} .
$$

If $\alpha\left(g_{1}, g_{2}\right) \equiv 0(\bmod 2 \pi)$ for all $g_{1}, g_{2}$, it is a genuine representation. In general it corresponds to a twist of the equivalence relations (13), (14) by a phase factor determined by $\alpha$.

Since $\alpha\left(g_{1}, g_{2}\right)$ is a function depending on two group elements $\left(g_{1}, g_{2}\right)$, it is called a two-cochain. The associativity of the product

$$
\begin{gathered}
{\left[\mathcal{U}\left(g_{0}\right) \mathcal{U}\left(g_{1}\right)\right] \mathcal{U}\left(g_{2}\right)=\mathcal{U}\left(g_{0}\right)\left[\mathcal{U}\left(g_{1}\right) \mathcal{U}\left(g_{2}\right)\right]} \\
\forall g_{0}, g_{1}, g_{2} \in \Gamma,
\end{gathered}
$$

implies that $\alpha$ has to be a two-cocycle; that is, its coboundary vanishes:

$$
\delta \alpha \equiv 0 \quad(\bmod 2 \pi) .
$$

The coboundary of $\alpha$ is defined as ${ }^{3}$

$$
\begin{aligned}
(\delta \alpha)\left(g_{0}, g_{1}, g_{2}\right)= & \alpha\left(g_{1}, g_{2}\right)-\alpha\left(g_{0} g_{1}, g_{2}\right)+\alpha\left(g_{0}, g_{1} g_{2}\right) \\
& -\alpha\left(g_{0}, g_{1}\right)(-1)^{c\left(g_{2}\right)} .
\end{aligned}
$$

Note that we have $\mathbf{Z}_{2}$ graded the usual definition of coboundary operator for group cohomology.

To fully determine a compactification we need to specify the two-cochain $\alpha$. But not all different assignments of $\alpha$ result in physically different compactifications. If we simply shift $\mathcal{U}(g)$ by a phase

$$
\mathcal{U}(g) \rightarrow \mathcal{U}(g) e^{i \beta(g)},
$$

the quotient conditions are not changed, but $\alpha$ will be shifted by the coboundary of the one-cochain $\beta$ as

$$
\alpha \rightarrow \alpha+\delta \beta,
$$

\footnotetext{
${ }^{3}$ The coboundary of a $k$-cochain is defined by

$(\delta \omega)\left(g_{0}, \ldots, g_{k}\right)=\omega\left(g_{1}, \ldots, g_{k}\right)$

$$
\begin{aligned}
& +\sum_{l=1}^{k}(-1)^{l} \omega\left(g_{0}, \ldots, g_{l-1} g_{l}, \ldots, g_{k}\right) \\
& +(-1)^{k+1} \omega\left(g_{0}, \ldots, g_{k-1}\right)(-1)^{c\left(g_{k}\right)} .
\end{aligned}
$$
}

where

$$
(\delta \beta)\left(g_{0}, g_{1}\right)=\beta\left(g_{1}\right)-\beta\left(g_{0} g_{1}\right)+\beta\left(g_{0}\right)(-1)^{c\left(g_{1}\right)} .
$$

Hence for a given compactification, $\alpha$ is only defined up to the coboundary of a one-cochain; i.e., $\alpha$ is a two-cocycle defined in the second Hochschild cohomology $H^{2}(\Gamma, U(1))$.

We can always use the ambiguity (27) to choose an $\alpha$ in a given equivalence class such that

$$
\alpha(e, g)=\alpha(g, e)=\alpha\left(g^{-1}, g\right)=0, \forall g \in \Gamma,
$$

where $e$ is the unity in $\Gamma$. To do so, we first make a transformation (27) with $\beta(e)=\alpha(e, e)$ so that $\alpha(e, g)=\alpha(g, e)$ $=0$. Then we can make another transformation with $\beta(g)$ $=\alpha\left(g^{-1}, g\right) / 2$ in order to get $\alpha\left(g^{-1}, g\right)=0$. In the following we assume that Eq. (29) is satisfied; so we can set

$$
\mathcal{U}(e)=1 \text { and } \mathcal{U}\left(g^{-1}\right)=\mathcal{U}(g)^{\dagger}
$$

For the case of $\Gamma=\mathbf{Z}_{2 n}$ which is generated by $e$ and $a$ with $a^{2 n}=e$ and $c(a)=1$, the second Hochschild cohomology is $H^{2}\left(\mathbf{Z}_{2 n}, U(1)\right)=\mathbf{Z}_{2}$, corresponding to the choice of sign in $\mathcal{U}(a)^{2 n}= \pm 1$. For the compactification on a twotorus, the discrete group is $\mathbf{Z}^{2}$ and $H^{2}\left(\mathbf{Z}^{2}, U(1)\right)$ is $U(1)$. This phase factor was found to correspond to the background three-form field $C$ on the two-torus and the light-cone circle $[6,11]$. In general, $H^{2}(\Gamma, U(1))$ is the moduli space of certain background parameters for a compactification. For orientifolds not all possible choices of elements in $H^{2}(\Gamma, U(1))$ are physically acceptible because some choices may lead to anomalous theories. For instance, for the compactification of matrix model on $S^{1} \times S^{1} / \mathbf{Z}_{2}$ to give the type I theory [12], new fields have to be added for anomaly cancellation. For the compactification on the orientifold $T^{5} / \mathbf{Z}_{2}$, the anomalyfree condition eliminates certain choices of $\alpha[13,14]$.

For the case of orbifolds there is a close analogy with the motion of electrons in a background of magnetic fields. Because the wave function of an electron changes by a phase $e^{i \int_{\gamma} d y^{\mu} A_{\mu}(y)}$ when moving along a path $\gamma$, the translation operators $U(\gamma)$ do not commute; rather, $U\left(\gamma_{1}\right) U\left(\gamma_{2}\right)$ $=e^{i \theta} U\left(\gamma_{2}\right) U\left(\gamma_{1}\right)$, where $\theta$ is given by the magnetic flux passing through the closed region bounded by the sequence of movements: $\left[\gamma_{1}, \gamma_{2},-\gamma_{1},-\gamma_{2}\right]$. This interpretation suggests that the noncommutativity between $U\left(g_{1}\right)$ and $U\left(g_{2}\right)$ in matrix model compactifications is related to the integration of a certain background field over a closed region defined by the sequence of actions by $g_{1}, g_{2}, g_{1}^{-1}, g_{2}^{-1}$. For the case of compactification on a two-torus the background field is the two-form $B$ field in string theory $[6,11,15]$ or, by duality, the three-form $C$ field in M theory. The coboundary condition on $\alpha$, Eq. (23), means that the field strength $H$ $=d B$ vanishes. So physically $\alpha$ represents the Wilson line degrees of freedom for the $B$ field on two-cycles of the compactified space. 


\section{SOLUTIONS OF QUOTIENT CONDITIONS}

The quotient conditions can be solved by following Zumino's prescription [7]. The idea is the same as Ref. [4]. Since the state of any D0-brane or open string can be related to those in the fundamental region labeled by $e$, all the degrees of freedom reside in the entries $X_{(g, i)(e, j)}^{\mu}$ and $\Psi_{(g, i)(e, j)}^{\alpha}$. Let us denote them by $\left(A_{g}^{\mu}\right)_{i j}$ and $\left(\Theta_{g}^{\alpha}\right)_{i j}$; then, we can use Eq. (19) to express $X$ and $\Psi$ in terms of $A$ and $\Theta$. These expressions are the solutions of quotient conditions.

Consider the Hilbert space

$$
\mathcal{H}_{0}=\{|g, i\rangle: g \in \Gamma, i=1,2, \ldots, n\},
$$

with the inner product

$$
\left\langle g_{1}, i \mid g_{2}, j\right\rangle=\delta_{g_{1} g_{2}} \delta_{i j} .
$$

Then

$$
X_{\left(g_{1}, i\right)\left(g_{2}, j\right)}^{\mu}=\left\langle g_{1}, i\left|X^{\mu}\right| g_{2}, j\right\rangle
$$

represents the open string stretching from the $i$ th D-particle in the fundamental region labeled by $g_{1}$ to the $j$ th D-particle in the fundamental region labeled by $g_{2}$ if $\left\langle g_{1}, i \mid g_{2}, j\right\rangle=0$. If $\left\langle g_{1}, i \mid g_{2}, j\right\rangle=1$, it represents the position of the $i$ th D-particle in the fundamental region labeled by $g_{1}$. Later we will see that $\mathcal{H}_{0}$ corresponds to a trivial $U(n)$ bundle on the dual space. We may choose other Hilbert spaces to represent nontrivial bundles.

In the following we will omit the second part in $\mathcal{H}_{0}$; that is, we will set $n=1$. It is straightforward to put the factor of $|i\rangle$ back later. The state $|e\rangle$ will be denoted " $\rangle$ ', and is called the vacuum. The state $|g\rangle$ can then also be defined by $U(g)\rangle$. The inner product on $\mathcal{H}_{0}$ implies that

$$
\langle U(g)\rangle=\delta_{g e} .
$$

By shifting the phase of the vacuum we can make it real:

$$
\rangle *=
$$

Using the quotient condition (15), we find

$$
\begin{aligned}
\left.X^{\mu} U(g)\right\rangle & \left.=U(g) \Phi_{g}^{\mu}\left(X^{* c(g)}\right)\right\rangle \\
& \left.=U(g)\left[R(g)_{\nu}^{\mu} X^{\nu *^{c(g)}}+a(g)^{\mu}\right]\right\rangle .
\end{aligned}
$$

Let

$$
\left.\left.X^{\mu}\right\rangle=A^{\mu}(U)\right\rangle
$$

where

$$
A^{\mu}(U)=\sum_{g} A_{g}^{\mu} U(g),
$$

with $A_{g}^{\mu}=\operatorname{Re}\left(A_{g}^{\mu}\right)+i \operatorname{Im}\left(A_{g}^{\mu}\right) \in \mathbf{C}$. Using Eqs. (35) and (37), we find

$$
\left.\left.X^{\nu *^{c(g)}}\right\rangle=A^{\nu}(U)^{*^{c(g)}}\right\rangle
$$

so

$$
\begin{aligned}
\left.X^{\mu} U(g)\right\rangle= & \left.U(g) \Phi_{g}^{\mu}\left(A(U)^{*^{c(g)}}\right)\right\rangle \\
= & {\left[\sum_{g^{\prime}} U\left(g^{\prime}\right) \Phi_{g^{\prime}}^{\mu}\left(A(U)^{*^{c\left(g^{\prime}\right)}}\right) U\left(g^{\prime}\right)^{\dagger} P\left(g^{\prime}\right)\right] } \\
& \times U(g)\rangle
\end{aligned}
$$

where we have used the projection operator $P\left(g^{\prime}\right)$ defined by

$$
\left.\left.P\left(g^{\prime}\right) U(g)\right\rangle=\delta_{g^{\prime} g} U(g)\right\rangle .
$$

The last line in Eq. (40) is an operator independent of $g$ acting on $U(g)\rangle$ for an arbitrary $g \in \Gamma$. It follows that

$$
X^{\mu}=\sum_{g} U(g) \Phi_{g}^{\mu}\left(A(U)^{*^{c(g)}}\right) U(g)^{\dagger} P(g) .
$$

Using the identity

$$
P\left(g_{1}\right) P\left(g_{2}\right)=\delta_{g_{1} g_{2}} P\left(g_{2}\right),
$$

which follows from Eq. (41), we can express $X^{\mu}$ more concisely as

$$
X^{\mu}(A)=\widetilde{A}^{\nu}(\widetilde{U}) \widetilde{R}_{\nu}^{\mu}+\widetilde{a}^{\mu}
$$

where

$$
\begin{aligned}
\widetilde{R}_{\nu}^{\mu} & =\sum_{g} R(g)_{\nu}^{\mu} P(g), \\
\tilde{a}^{\mu} & =\sum_{g} a(g)^{\mu} P(g), \\
\widetilde{A}^{\nu}(\widetilde{U}) & =\sum_{g} \widetilde{U}(g) \widetilde{A}_{g}^{\nu}, \\
\widetilde{U}(g) & =\sum_{g^{\prime}} U\left(g^{\prime}\right) U(g)^{*^{c\left(g^{\prime}\right)}} U\left(g^{\prime}\right)^{\dagger} P\left(g^{\prime}\right), \\
\widetilde{A}_{g}^{\nu} & =\operatorname{Re}\left(A_{g}^{\nu}\right)+\widetilde{i} \operatorname{Im}\left(A_{g}^{\nu}\right), \\
\widetilde{i} & =i \sum_{g}(-1)^{c(g)} P(g) .
\end{aligned}
$$

Equation (44) is the most general solution of $X$ on the Hilbert space $\mathcal{H}_{0}$.

Similarly, let

$$
\left.\left.\Psi^{\alpha}\right\rangle=\Theta^{\alpha}(U)\right\rangle,
$$

where

$$
\Theta^{\alpha}(U)=\sum_{g} \Theta_{g}^{\alpha} U(g)
$$

then, 


$$
\Psi^{\alpha}=\widetilde{\Theta}^{\beta} \widetilde{\Lambda}_{\beta}^{\alpha},
$$

where

$$
\begin{gathered}
\widetilde{\Lambda}_{\beta}^{\alpha}=\sum_{g} \Lambda(g)_{\beta}^{\alpha} P(g), \\
\widetilde{\Theta}^{\alpha}(\widetilde{U})=\sum_{g} \widetilde{U}(g) \widetilde{\Theta}_{g}^{\alpha}, \\
\widetilde{\Theta}_{g}^{\alpha}=\operatorname{Re}\left(\widetilde{\Theta}_{g}^{\alpha}\right)+\widetilde{i} \operatorname{Im}\left(\widetilde{\Theta}_{g}^{\alpha}\right) .
\end{gathered}
$$

For $n>1, A_{g}^{\mu}$ and $\Theta_{g}^{\alpha}$ become $n \times n$ matrices which are constrained by the Hermiticity of $X$ and $\Psi$.

To check directly that the quotient conditions (19) are satisfied by this solution, we can use the following relations

$$
\begin{aligned}
\widetilde{R}_{\nu}^{\mu} U(g) & =U(g) R(g)_{\sigma}^{\mu} \widetilde{R}_{\nu}^{\sigma}, \\
\tilde{a}^{\mu} U(g) & =U(g)\left[R(g)_{\nu}^{\mu} \widetilde{a}^{\nu}+a(g)^{\mu}\right], \\
\widetilde{U}(g) U\left(g^{\prime}\right) & =U\left(g^{\prime}\right) \widetilde{U}(g)^{*^{c\left(g^{\prime}\right)},} \\
\widetilde{U}(g) \mathcal{U}\left(g^{\prime}\right) & =\mathcal{U}\left(g^{\prime}\right) \widetilde{U}(g), \\
\widetilde{i} U(g) & =U(g) \tilde{i}^{*(g)}, \\
\widetilde{i} \mathcal{U}(g) & =\mathcal{U}(g) \tilde{i} .
\end{aligned}
$$

To derive them we used Eqs. (8),(9) and

$$
P(g) U\left(g^{\prime}\right)=U\left(g^{\prime}\right) P_{g^{\prime-1} g},
$$

which follows from Eq. (41).

After the quotient conditions are solved, the matrix model is only concerned with $\widetilde{U}, \widetilde{R}, \tilde{a}$, and $\widetilde{i}$, which form a closed algebra:

$$
\begin{gathered}
\widetilde{U}\left(g_{1}\right) \widetilde{U}\left(g_{2}\right)=e^{\tilde{i} \alpha\left(g_{2}, g_{1}\right)} \widetilde{U}\left(g_{2} g_{1}\right), \\
{\left[\widetilde{R}_{\nu}^{\mu}, \tilde{a}^{\sigma}\right]=\left[\widetilde{R}_{\nu}^{\mu}, \widetilde{i}\right]=\left[\tilde{a}^{\mu}, \widetilde{i}\right]=0,} \\
\widetilde{R}_{\nu}^{\mu} \widetilde{U}(g)=\widetilde{U}(g) \widetilde{R}_{\sigma}^{\mu} R(g)_{\nu}^{\sigma}, \\
\widetilde{a}^{\mu} \widetilde{U}(g)=\widetilde{U}(g)\left[\widetilde{R}_{\nu}^{\mu} a(g)^{\nu}+\tilde{a}^{\mu}\right], \\
\widetilde{i}^{2}=1, \tilde{i} \widetilde{U}(g)=\widetilde{U}(g) \widetilde{i}^{c(g)} .
\end{gathered}
$$

Equations (64)-(68) are derived from Eqs. (45),(46), (48),(50). Now that we have obtained the algebra of $\widetilde{U}, \widetilde{R}, \tilde{a}$, and $\tilde{i}$, we can forget about $U$. Since $\widetilde{U}(g)\rangle$ $=U(g)\rangle, \mathcal{H}_{0}$ can also be written as $\left.\{\widetilde{U}(g)\rangle\right\}$. The functional (34) which determines the inner product on $\mathcal{H}_{0}$ can be viewed as a functional on $\widetilde{U}$ :

$$
\langle\widetilde{U}(g)\rangle=\delta_{g e} .
$$

Recall that in Sec. III we have assumed that $U(g)$ is a matrix of numbers and thus it commutes with any complex number (times the unit matrix). In the solution of $X$, the operators $\widetilde{U}, \widetilde{R}, \tilde{a}$, and $\widetilde{A}$ are also matrices of numbers; so they all commute with the complex number $i$. Thus $U(g)$ commutes with $i$ while $\mathcal{U}(g)$ commutes with $\tilde{i}$ for all $g \in \Gamma$. On the other hand, $\mathcal{U}(g)$ does not commute with $i$ if $c(g)$ $=1$, and $\widetilde{U}(g)$ does not commute with $\tilde{i}$ if $c(g)=1$. In fact one can find another representation in which the states are $\{\mathcal{U}(g)\rangle\}$ where $\tilde{i}$ is represented as $i$ times the unit matrix and $i$ is represented by the nontrivial matrix which represents $\tilde{i}$ on the states $\{\widetilde{U}(g)\rangle\}$. Furthermore, the algebra of $\widetilde{U}$ is the same as the algebra of right multiplication on the states $\{\mathcal{U}(g)\rangle\}$ by $\mathcal{U}$. Roughly speaking, the algebra of $\mathcal{U}$ is dual to the algebra of $\widetilde{U}$ and $i$ is dual to $\tilde{i}$. This duality corresponds to the symmetry of charge conjugation on D-branes.

The solution (44) of $X$ appears to be of the same form as a covariant derivative on a quantum space. The function $\widetilde{A}(\widetilde{U})$ plays the role of gauge fields and both $\tilde{a}$ and $\widetilde{R}$ play the role of derivatives. The algebra of $\widetilde{U}$, Eq. (64), is viewed as the algebra of functions on the base space of the matrix model as a gauge field theory. For toroidal compactifications, $X$ indeed turns out to be the usual covariant derivative on the dual torus. Note that the operator $\widetilde{R}$ is also a generalized derivative on a quantum space. For instance the covariant derivative on the space of $\mathbf{Z}_{n}$ (discrete $n$ points) is given by an operator of the form (44) without the last term $\tilde{a}$.

\section{NONCOMMUTATIVE GEOMETRY}

For completeness we briefly review in this section elements of noncommutative geometry [16]. The Riemannian structure of a quantum space is encoded in the spectral triple $(\mathcal{A}, \mathcal{H}, \mathcal{D})[16]$, where $\mathcal{A}$ is the algebra of functions on the quantum space, $\mathcal{H}$ is the Hilbert space on which $\mathcal{A}$ is realized as an operator algebra, and $\mathcal{D}$ is the so-called "Dirac operator" which is a self-adjoint operator on $\mathcal{H}$. The Dirac operator is used to define the differential calculus on the quantum space.

The naive definition for integration on a quantum space is just the trace over $\mathcal{H}$. While $\mathcal{H}$ can be infinite dimensional, the definition of integration may have to be regulated as

$$
\int f=\lim _{\Lambda \rightarrow \infty} \frac{\operatorname{Tr}_{\mathcal{H}}\left(f e^{-|\mathcal{D}|^{2} / \Lambda^{2}}\right)}{\operatorname{Tr}_{\mathcal{H}}\left(e^{-|\mathcal{D}|^{2} / \Lambda^{2}}\right)},
$$

which is reminiscent of the regularization in quantum field theory. The general definition of integration on a quantum space is given by the Dixmier trace [16]. The functional (69) is generically different from the integration for orientifolds although they always agree for manifolds and orbifolds. The most important property of integration is cyclicity, that is,

$$
\int U^{\dagger} f U=\int f
$$


for a (reasonably well-behaved) unitary operator $U$ on $\mathcal{H}$. The cyclity is important because it enables us to find a gauge-invariant action for a gauge theory on a quantum space.

A differential $k$-form on a quantum space is a formal expression like

$$
\rho=\sum_{i} a_{0}^{i} d a_{1}^{i} \cdots d a_{k}^{i}
$$

where $a_{j}^{i}, j=0,1, \ldots, k$ are elements of $\mathcal{A}$. An element of $\mathcal{A}$ can be multiplied to $\rho$ from the left or from the right. If it is multiplied from the right, one can use the Leibniz rule to rewrite the product in the standard form (72). Denote the set of $k$-forms as $\Omega^{k} \mathcal{A}$. We define $\Omega \mathcal{A}$ as $\oplus_{k} \Omega^{k} \mathcal{A}$ on which the exterior derivative $d$ acts. We impose on $\Omega \mathcal{A}$ the nilpotency condition

$$
d^{2}=0
$$

and the Leibniz rule

$$
d\left(\rho_{1} \rho_{2}\right)=\left(d \rho_{1}\right) \rho_{2} \pm \rho_{1}\left(d \rho_{2}\right),
$$

where the sign depends on whether $\rho_{1}$ is an even form $(+)$ or an odd form $(-)$.

For a given representation $\pi$ of $\mathcal{A}$ on $\mathcal{H}$ we can extend it to $\Omega \mathcal{A}$ by defining

$$
\pi(\rho)=\sum_{i} a_{0}^{i}\left[\mathcal{D}, a_{1}^{i}\right] \cdots\left[\mathcal{D}, a_{k}^{i}\right]
$$

for a $k$-form expressed as in Eq. (72). To simplify our notation here and below we simply write $a$ to stand for $\pi(a)$. The inner product of two differential $k$-forms $\rho_{1}, \rho_{2}$ can be defined as

$$
\left\langle\rho_{1} \mid \rho_{2}\right\rangle=\int \pi\left(\rho_{1}\right)^{\dagger} \pi\left(\rho_{2}\right) .
$$

The operator algebra on $\mathcal{H}$ induces a differential calculus $\Omega(\mathcal{A})$ through the representation $\pi$ by defining

$$
\Omega(\mathcal{A})=\oplus_{k}\left(\Omega^{k} \mathcal{A} / J_{k}\right),
$$

where

$$
J_{k}=\left.\operatorname{ker} \pi\right|_{\Omega^{k} A}+d\left(\left.\operatorname{ker} \pi\right|_{\Omega^{k-1} A}\right) .
$$

Therefore, two differential forms $\rho_{1}, \rho_{2} \in \Omega^{k} \mathcal{A}$ are equivalent if $\pi\left(\rho_{1}-\rho_{2}\right)=0$ or if $\left(\rho_{1}-\rho_{2}\right)=d \rho$ for a certain differential form $\rho \in \Omega^{k-1} \mathcal{A}$ with $\pi(\rho)=0$. However, the representation $\pi$ does not respect this equivalence relation. We should therefore define another representation $\hat{\pi}$ which is $\pi$ preceded by a projection onto the subspace of $\Omega^{k} \mathcal{A}$ orthogonal to $J_{k} \cdot{ }^{4}$

\footnotetext{
${ }^{4}$ Two differential forms are orthogonal if their inner product vanishes.
}

In our case of interest, the Dirac operator is of the form

$$
\mathcal{D}=\gamma^{\mu} \mathcal{D}_{\mu}
$$

where the $\gamma^{\mu}$,s are the usual gamma matrices which commute with $\mathcal{D}$ and $\mathcal{A}$, and they satisfy $\left\{\gamma^{\mu}, \gamma^{\nu}\right\}=2 \eta^{\mu \nu}$. This type of Dirac operators were discussed in detail in Ref. [9], where it was shown that if the conditions

$$
\begin{aligned}
& {\left[\mathcal{D}_{\mu}, \mathcal{D}_{\nu}\right]=0,} \\
& \pi\left(J_{2}\right)=\pi(\mathcal{A})
\end{aligned}
$$

are satisfied, then

$$
\hat{\pi}(d \rho)=\frac{1}{2} \gamma^{\mu \nu}\left(\left[\mathcal{D}_{\mu}, \rho_{\nu}\right]-\left[\mathcal{D}_{\nu}, \rho_{\mu}\right]\right)
$$

for any differential one-form $\rho$, where $\hat{\pi}(\rho)=\gamma^{\mu} \rho_{\mu}$ and $\gamma^{\mu \nu}=\frac{1}{2}\left[\gamma^{\mu}, \gamma^{\nu}\right]$. The condition for Eq. (81) to be satisfied is that any element in $\pi(\mathcal{A})$ can be written as

$$
\sum_{i} a_{i}\left[\eta^{\mu \nu} \mathcal{D}_{\mu} \mathcal{D}_{\nu}, b_{i}\right]
$$

for some $a_{i}, b_{i} \in \mathcal{A}$.

Let $A$ denote the gauge field in a Yang-Mills theory. The gauge field strength is $F=d A+A^{2}$. We define a dressed Dirac operator

$$
\widetilde{\mathcal{D}}=D+\hat{\pi}(A)
$$

where $A$ is a one-form $\sum_{i} a_{i} d b_{i}$ for some $a_{i}, b_{i} \in \mathcal{A}$ and $\hat{\pi}(A)=\sum_{i} a_{i}\left[\mathcal{D}, b_{i}\right]$. Then it follows from Eq. (82) that

$$
\hat{\pi}(F)=\frac{1}{2} \gamma^{\mu \nu}\left[\widetilde{\mathcal{D}}_{\mu}, \widetilde{\mathcal{D}}_{\nu}\right]
$$

The action for a Yang-Mills theory on a quantum space is defined to be [16]

$$
S_{Y M}=\frac{1}{g_{Y M}^{2}}\langle F \mid F\rangle .
$$

The gauge field can be coupled to a fermionic matter field $\Psi$ by adding the term [9]

$$
S_{f}=\langle\Psi|\widetilde{\mathcal{D}}| \Psi\rangle
$$

and the total action is

$$
S=S_{Y M}+S_{f}
$$

It gives a super Yang-Mills (SYM) theory on a quantum space for our matrix variables $X$ and $\Psi$ if Eq. (85) is satisfied [9]. If Eq. (85) does not hold, one can still define a YangMills theory with matter on a quantum space by the action (88), but then there will be new terms compared with the standard SYM action. 


\section{QUANTUM SPACE}

Interpreting the matrix model compactified on a quotient space as a gauge field theory on a quantum space, we need to specify the spectral triple $(\mathcal{A}, \mathcal{H}, \mathcal{D})$ for the base space. First, the algebra of functions $\mathcal{A}$ on the base space is the algebra of $\widetilde{U}^{5}{ }^{5}$ because the gauge fields $\widetilde{A}^{\mu}(\widetilde{U})$ are functions of $\widetilde{U}$ for a trivial bundle. We take the bare Dirac operator to be

$$
\mathcal{D}=\gamma_{\mu} X^{\mu}\left(A_{0}\right)
$$

where $A_{0}^{\mu}$ are generic constant diagonal matrices. This operator defines the calculus and it has the property of flat connection

$$
\left[X_{\mu}\left(A_{0}\right), X_{\nu}\left(A_{0}\right)\right]=0
$$

as in Eq. (80).

Given the solution $X^{\mu}\left(A_{0}\right)$ of the quotient conditions,

$$
X^{\mu}=\sum_{i} a_{i}(\widetilde{U}) X^{\mu}\left(A_{0}\right) b_{i}(\tilde{U})
$$

is also a solution of the quotient condition (19) because $\widetilde{U}$ commutes with $\mathcal{U}$. If $\sum_{i} a_{i} b_{i}=1$, then Eq. (91) can also be written as

$$
X^{\mu}=X^{\mu}\left(A_{0}\right)+\hat{\pi}\left(A^{\prime}\right)^{\mu}
$$

where $A^{\prime}=\sum_{i} a_{i} d b_{i}$ is a generic one-form field. Thus we see that the interpretation of $X$ as a covariant derivative, i.e., the partial derivative plus a one-form field, is a very natural result of the fact that $X$ is a solution of the quotient condition. Conversely, we may take this as a guiding principle for the study of gauge theories on noncommutative space. We may even view the quotient condition as the fundamental physical reason for the appearance of covariant derivatives in gauge theories.

It follows that for generic $A_{0}$ the dressed Dirac operator is

$$
\widetilde{\mathcal{D}}=\gamma_{\mu} X^{\mu}(A)
$$

for an arbitrary function $A$ of $\widetilde{U}$. Since $\mathcal{H}$ in the spectral triple is defined to be the Hilbert space on which both $\mathcal{A}$ and $\mathcal{D}$ are realized as operators, it should consist of both $\mathcal{H}_{0}$ and the space of Dirac spinors in $(9+1)$ dimensions.

Assuming Eq. (82), we find

$$
\hat{\pi}(F)=\frac{1}{2} \gamma^{\mu \nu} F_{\mu \nu},
$$

where

$$
F_{\mu \nu}=\left[X_{\mu}, X_{\nu}\right]
$$

\footnotetext{
${ }^{5} \mathrm{~A}$ generic element in this algebra is of the form $\Sigma_{g} a_{g} \widetilde{U}(g)$ with
} $a(g) \in \mathbf{C}$.
Then Eq. (88) gives precisely the SYM action for the matrix model. The matrix model compactified on the orientifold of flat superspace over $\Gamma$ is thus identified with the SYM theory on the quantum space $(\mathcal{A}, \mathcal{H}, \mathcal{D})$.

Define a set of one-forms by

$$
\xi_{g}=\widetilde{U}(g)^{\dagger} d \widetilde{U}(g) .
$$

The Leibniz rule implies that

$$
\xi_{g^{\prime}} \widetilde{U}(g)=\widetilde{U}(g)\left(\xi_{g g^{\prime}}-\xi_{g}\right)
$$

Using

$$
\pi\left(\xi_{g}\right)=\gamma_{\mu} \widetilde{R}_{\nu}^{\mu}\left[\left(R(g)_{\sigma}^{\nu}-\delta_{\sigma}^{\nu}\right) A_{0}^{\sigma}+a(g)^{\nu}\right]
$$

according to Eq. (77), the differential calculus on the quantum space satisfies the following commutation relations:

$$
\left\{\xi_{g}, \xi_{g^{\prime}}\right\}=0
$$

Then it also follows that

$$
\xi_{g}^{2}=d \xi_{g}=0
$$

In particular, if $R(g)_{\nu}^{\mu}=\delta_{\nu}^{\mu}$ for a certain $g \in \Gamma$, then

$$
\left[\xi_{g}, \widetilde{U}_{g^{\prime}}\right]=0, \forall g^{\prime} \in \Gamma
$$

In the above we have chosen a particular representation for $\Gamma$ which is the projective regular representation, but we may choose other representations. A different representation in general means a different background. If we take the Hilbert space $\mathcal{H}$ as above, the gauge fields $A$ are defined as functions of $\widetilde{U}$ 's, which are functions on the quantum space. This means that it lives on a trivial bundle. The definition of a generic (twisted) bundle on a quantum space is that the set of sections on a twisted bundle is a projective module of $\mathcal{A}$ [16]. ${ }^{6}$ This definition follows from the classical theorem that all locally trivial finite dimensional complex vector bundles over a compact space $\mathcal{M}$ are in one-to-one correspondence with projective modules over the algebra of continuous functions on $\mathcal{M}$. For any projective module of $\mathcal{A}$ we can define a right multiplication of $\mathcal{A}$ on it. From our example of the regular representation, we see that we should view the left multiplication of $\mathcal{U}(g)$ as the right multiplication of $\widetilde{U}(g)$ for a generic projective representation, while the left multiplication of $\widetilde{U}(g)$ is in general not defined. Sections on the bundle of adjoint representations are represented by operators on the projective module which commute with all $\mathcal{U}(g)$ 's. Indeed the space of solutions of $X$ to the quotient conditions is the same space of operators on the Hilbert space commuting with all $\mathcal{U}(g)$ 's.

\footnotetext{
${ }^{6} \mathrm{~A}$ projective module is a direct summand of a (finitely generated) free module.
} 


\section{RESIDUAL GAUGE SYMMETRY}

Before the quotient conditions are imposed, the gauge group is $U(N)$ where $N$ is the dimension of the Hilbert space. After the quotient conditions are imposed, a gauge transformation by $\hat{g} \in U(N)$,

$$
X^{\mu} \rightarrow \hat{g}^{\dagger} X^{\mu} \hat{g}
$$

survives the quotient conditions (19) only if

$$
\hat{g} \mathcal{U}(h) \hat{g}^{\dagger}=\mathcal{U}(h) e^{i \beta(h)}
$$

or, equivalently,

$$
\hat{g} U(h)\left(\hat{g}^{\dagger}\right)^{c(h)}=e^{i(-1)^{c(h)} \beta(h)} U(h)
$$

for a certain function $\beta$ on $\Gamma$. Note that its consistency with the algebra of $U$, Eq. (21), implies that the coboundary of $\beta$, Eq. (28),

$$
\delta \beta\left(g_{0}, g_{1}\right) \equiv 0(\bmod 2 \pi)
$$

that is, $\beta$ has to be a one-cocycle. For $g_{0}=e$, Eq. (105) implies that $\beta(e)=0$, which is compatible with Eq. (30). Actually, $\beta$ is only defined up to an exact one-cochain because if we shift $\hat{g}$ by a phase $e^{i \omega}, \beta$ transforms as

$$
\beta \rightarrow \beta-\delta \omega,
$$

where $\delta \omega(g)$ is given by

$$
\delta \omega(g)=\omega-\omega(-1)^{c(g)} .
$$

Thus $\beta$ is defined as an element in $H^{1}(\Gamma, U(1))$. is

According to Eq (103), the transformation of $\widetilde{U}(h)$ by $\hat{g}$

$$
\hat{g} \widetilde{U}(h) \hat{g}^{\dagger}=e^{\tilde{i}(-1)^{c(h)} \beta(h)} \widetilde{U}(h),
$$

which induces a transformation on $A$ by

$$
\widetilde{A}_{h}^{\mu} \rightarrow e^{\tilde{i}(-1)^{c(h)} \beta(h)} \widetilde{A}_{h}^{\mu} .
$$

For different Hilbert space $\mathcal{H}$ we have different elements $\hat{g}$ satisfying Eq. (103) and thus different $\beta$ 's.

Obviously, if $\hat{g}$ is an arbitrary function of $\widetilde{U}$, then Eq (103) is satisfied with $\beta=0$. In fact these functions of $\widetilde{U}$ include all the $\hat{g}$ 's with $\beta=0$ for the Hilbert space $\mathcal{H}_{0}$; otherwise, Eq. (44) would not have been the most general solution of $X$. If $\hat{g}$ is a function of $\widetilde{U}$, then it is interpreted as a local gauge transformation on the dual quantum space. On the other hand, if $\beta(g) \neq 0$ for some $g \in \Gamma, \hat{g}$ indicates a global symmetry. Note that the existence of $\hat{g}$ depends on the choice of the Hilbert space.

\section{EXAMPLES}

In this section we give a few examples to show how the above formulation works for practical cases. For complete- ness we also include examples of manifold and orbifold as well as orientifold. More examples can be found in [7].

\section{A. Torus}

A torus $T^{d}$ is a quotient space $\mathbf{R}^{d} / \mathbf{Z}^{d}$. For simplicity consider a rectangular torus. The action of the group $\Gamma=\mathbf{Z}^{d}$ on $\mathbf{R}^{d}$ is

$$
\Phi_{\mathbf{m}}^{i}(X)=X^{i}+2 \pi m_{i} R_{i}, \quad i=1, \ldots, d,
$$

where $\mathbf{m}=\left(m_{1}, \ldots, m_{d}\right), m_{i} \in \mathbf{Z}$, is an element in $\Gamma$. This is a manifold, and so $c(g)=0$ for all $g \in \Gamma$. The algebra of $U$ is generically given by [6]

$$
U(\mathbf{m}) U(\mathbf{n})=e^{2 i \alpha(\mathbf{m}, \mathbf{n})} U(\mathbf{n}) U(\mathbf{m}),
$$

where $\alpha(\mathbf{m}, \mathbf{n})=\theta^{\mu \nu} m_{\mu} n_{\nu}$ and $\theta$ is an antisymmetric $d \times d$ matrix of real numbers. The algebra of $\widetilde{U}$ is then

$$
\tilde{U}(\mathbf{m}) \tilde{U}(\mathbf{n})=e^{-2 i \alpha(\mathbf{m}, \mathbf{n})} \tilde{U}(\mathbf{n}) \tilde{U}(\mathbf{m}),
$$

which is viewed as the algebra of functions on the dual quantum torus. A large amount of literature can be found on this subject (see, for instance, Refs. [6,11,15,17-23]).

The solution of $X$ is that it is a covariant derivative on the quantum torus. We find $\widetilde{R}_{j}^{i}=\delta_{j}^{i}, \widetilde{a}^{i}=-i \partial_{i}$, and $\tilde{i}=i$, where $\left[\partial_{i}, \widetilde{U}(\mathbf{m})\right]=i m_{i} \widetilde{U}(\mathbf{m})$ just like ordinary derivatives. For trivial bundles $X^{i}=-i \partial_{i}+A^{i}(\widetilde{U})$.

Integration over the quantum torus is given by

$$
\langle\widetilde{U}(\mathbf{m})\rangle=\prod_{i=1}^{d} \delta_{m_{i}, 0}
$$

up to a normalization. Equation (85) holds; so the gaugeinvariant action (88) is of the same form as the SYM action on a classical torus. The only new ingredient is that the base space is not commutative. The differential calculus is almost classical:

$$
[\widetilde{U}(\mathbf{m}), \xi(\mathbf{n})]=0 .
$$

Twisted bundles on the quantum torus are defined to be projective modules of the algebra of $\widetilde{U}$. Classically one can describe a twisted bundle by twisted boundary conditions on sections of the bundle. For instance, for a section of the bundle in the fundamental representation of $U(n)$, we have the boundary conditions

$$
\phi\left(\sigma_{1}, \ldots, \sigma_{j}+2 \pi, \ldots, \sigma_{d}\right)=\Omega_{j} \phi\left(\sigma_{1}, \ldots, \sigma_{j}, \ldots, \sigma_{d}\right)
$$

for $j=1, \ldots, d$, and $\Omega$ is an $n \times n$ matrix of functions of $\left(\sigma_{1}, \ldots, \sigma_{j-1}, \sigma_{j+1}, \ldots, \sigma_{d}\right)$ which specifies the gauge transformation of the $n$-vector $\phi$ when $\sigma_{j}$ is shifted by a whole cycle. Note that the sections $\phi$ are in general not functions well defined on the torus; otherwise, they are sections of the trivial bundle. Rather, the sections are functions on the infinite plane with coordinates $\sigma_{j}$ viewed as the covering space of the torus. 
Here we make a general remark about twisted bundles on quantum spaces. In the classical case, sections of a twisted bundle have the following properties. First, one can multiply a function well defined on the torus from the right, because this will not spoil the boundary condition. Second, one can multiply a section of the adjoint representation from the left for the same reason. Third, there is a definition of inner product for two sections which gives a function well defined on the torus. For the fundamental representation the inner product of $\phi$ and $\phi^{\prime}$ is just $\sum_{i=1}^{n} \phi_{i}^{\dagger} \phi_{i}^{\prime}$. In the quantum case, all three properties are preserved. The multiplication by functions of $\widetilde{U}$ from the right is defined to be the multiplication by functions of $\mathcal{U}$ from the left, which can always be defined since the $\mathcal{U}$ 's are always operators on the Hilbert space on which the quotient conditions are defined. The other two properties are preserved simply because the twisted boundary conditions for the quantum case are formally the same as for the classical case. An explicit construction of the bundle by imposing twisted boundary conditions on sections is given in Ref. [17]. Projective modules on quantum spaces or for any $\Gamma$ being a discrete Abelian group are discussed in detail in Ref. [24].

As mentioned in Sec. VII, we need to impose gauge invariance conditions on physical states for each element $\hat{g}$ satisfying Eq. (103) with $\beta=0$. For the twisted bundle on the quantum torus $T_{\theta}^{2}$, sections of the adjoint representations are generated by two sections $Z_{1}$ and $Z_{2}$, both satisfying Eq. (103) with $\beta=0$. Since [6]

$$
Z_{1} Z_{2}=e^{i 2 \pi \theta^{\prime}} Z_{2} Z_{1}
$$

where $\theta^{\prime}=(b-\theta a) /(n-\theta m)$ and $a, b$ are integers satisfying $a n-b m=1$, they induce the transformations

$$
X_{i}=-i 2 \pi R_{i} D_{i}+A_{i}\left(Z_{1}, Z_{2}\right)
$$

where $D_{i}$ is the covariant derivative on $T_{\theta}^{2}$. Let the operator conjugate to the constant part of $X_{i}$ be denoted $E_{i}$, and the operator shifting the phase of $Z_{i}$ be denoted $P_{i}^{\prime}$. Then corresponding to the gauge invariance under conjugations by $Z_{i}$, the gauge transformation operator

$$
\exp \left(i 2 \pi \frac{R_{i}}{n-\theta m}\left(E_{i}-\theta^{\prime} \epsilon_{i j} P_{j}^{\prime}\right)\right)=\mathbf{1}
$$

when acting on a physical state. Since the phase of $Z_{i}$ is defined up to $2 \pi$, the eigenvalues of $P_{i}^{\prime}$ are integers. Let $P_{i}^{\prime}=m_{i} \in \mathbf{Z}$; then, the gauge invariance means

$$
E_{i}=\frac{n-\theta m}{R_{i}}\left(n_{i}+\theta^{\prime} \epsilon_{i j} m_{j}\right)
$$

for integer $n_{i}$. This result differs from that in [17], for which proper corrections were pointed out in [25] and [26].

$$
\text { B. } A_{n-1}
$$

The asymptotically local Euclidean (ALE) space $A_{n-1}$ is the quotient space $\mathbf{R}^{4} / \mathbf{Z}_{n}$. The physics of D-branes on $A_{n-1}$ was first described in Ref. [2], which in fact demonstrated the general principle for D-branes on all kinds of quotient spaces. Here we discuss this case as a simple example.

The action of $\Gamma=\mathbf{Z}_{n}$ is generated by

$$
Z_{i} \rightarrow U^{\dagger} Z_{i} U=\Phi_{i}(Z)=e^{i 2 \pi / n} Z_{i},
$$

where $Z_{i}=X_{i}+i X_{i+2}$ for $i=1,2$ and $m \in \mathbf{Z}_{n}$. This is an orbifold, and so $c(m)=0$. For a projective representation of $\mathbf{Z}_{n}, U^{n}=e^{i \theta}$ for some $\theta \in \mathbf{R}$. By a change of phase of $U$ one can always set $\theta=0$. This means that $H^{2}(\Gamma, U(1))$ is trivial and we can set $\alpha=0$. The algebra of functions on the quantum space is $\mathcal{A}=\mathbf{Z}_{n}$.

The bare Dirac operator on this quantum space is $\widetilde{R}$ [16], which satisfies

$$
\widetilde{R} U=e^{i 2 \pi / n} U \widetilde{R} .
$$

Then a generic differential one-form $A$ has $\hat{\pi}(A)=f \widetilde{R}$ for some $f \in \mathcal{A}$. It follows that the dressed Dirac operator is also of the form $\widetilde{\mathcal{D}}=f \widetilde{R}$. Since $\tilde{a}=0$, for a trivial bundle the solution of $Z$ is

$$
Z=A(U) \widetilde{R},
$$

which is understood as a covariant derivative on $\mathbf{Z}_{n}$. Note that $\widetilde{U}=U$ in this case.

In the regular representation (for the trivial bundle), $U$ and $\widetilde{R}$ are $n \times n$ matrices. For instance we can choose

$$
U_{i j}=e^{i 2 j \pi / n} \delta_{i j}, \quad \widetilde{R}_{i j}=\delta_{(i+1) j} .
$$

Here $U$ is diagonal and it generates the algebra of functions on $n$ points, which is represented as a diagonal $n \times n$ matrix. The commutator of $\widetilde{R}$ with a diagonal matrix $f$ $=\operatorname{diag}\left(f_{1}, \ldots, f_{n}\right)$ is $\left(f_{i+1}-f_{i}\right) \widetilde{R}_{i j}$, with the factor of 
$\left(f_{i+1}-f_{i}\right)$ as one would intuitively expect for a derivative for a discrete space. Another choice of representation is

$$
U_{i j}=\delta_{(i+1) j}, \quad \widetilde{R}_{i j}=e^{-i 2 j \pi / n} \delta_{i j} .
$$

In this basis $U$ is off diagonal, corresponding to the interpretation that it represents the open strings stretched between the $n$ images of D-branes in the covering space. These two representations are in this sense dual to each other. The latter representation is more natural in terms of D0-branes in the original compactified spacetime, while the former representation is more natural when considering the quantum base space $\mathbf{Z}_{n}$ on which the matrix model is viewed as a gauge theory.

It is easy to check that Eq. (82) is satisfied; hence, the action (88) agrees with the matrix model action (SYM action). The differential calculus is defined by

$$
U \xi=e^{-i 2 \pi / n} \xi U,
$$

where $\xi=U^{\dagger} d U$. Projective modules on $\mathbf{Z}_{n}$ is discussed in Ref. [24] and can also be obtained by the method given in Ref. [17].

\section{Gauging world sheet parity}

Now we consider gauging only the world sheet parity. The quotient condition for this case is

$$
U^{\dagger} X^{\mu} U=X^{\mu *} .
$$

The algebra of $U$ is defined by

$$
U U^{*}=\epsilon \mathbf{1}
$$

where $\epsilon= \pm 1$. The choice of $\epsilon$ corresponds to the choice of an element in $H^{2}\left(\mathbf{Z}_{2}, U(1)\right)=\mathbf{Z}_{2}$. A unitary transformation of $X$,

$$
X \rightarrow V^{\dagger} X V
$$

is equivalent to a transformation on $U$ by

$$
U \rightarrow V U V^{T}
$$

where $T$ denotes transposition. It is straightforward to show that there are only three inequivalent two-dimensional representations of $U$ :

$$
U=\left(\begin{array}{ll}
1 & 0 \\
0 & 1
\end{array}\right),\left(\begin{array}{ll}
0 & 1 \\
1 & 0
\end{array}\right),\left(\begin{array}{cc}
0 & -i \\
i & 0
\end{array}\right),
$$

where the first two choices are for $\epsilon=1$ and the last choice is for $\epsilon=-1$. It is amusing that although the first two cases are not related by unitary transformations, they are equivalent for the purpose of the matrix model; i.e., there exists a map between matrix variables for the two choices so that they give the same action for the matrix model [27]. The reason why we are interested in two-dimensional representations of $U$ is that we are interested in projective regular representations of $\Gamma=\mathbf{Z}_{2}$.

Using the solution (44) we find immediately that

$$
X=\left(\begin{array}{ll}
A & B \\
B^{\dagger} & D
\end{array}\right),
$$

where $D^{*}=A$ and $B^{T}=\epsilon B$.

$$
\text { D. } S^{1} \times S^{1} / Z_{2}
$$

In this example we consider the space $S^{1} \times S^{1} / \mathbf{Z}_{2}$ which is related to the heterotic matrix strings [29-31,12,32-35,7].

The quotient conditions are

$$
\begin{aligned}
U_{i}^{\dagger} X_{j} U_{i} & =X_{j}+2 \pi \delta_{i j} R_{j}, i, j=1,2, \\
U_{3}^{\dagger} X_{1} U_{3} & =-X_{1}^{*}, \\
U_{3}^{\dagger} X_{2} U_{3} & =X_{2}^{*} .
\end{aligned}
$$

The algebra of $U$ is determined by a phase $q$ and two signs $\epsilon_{1}, \epsilon_{2}$ as [7]

$$
\begin{aligned}
& U_{1} U_{2}=q U_{2} U_{1}, \\
& U_{1} U_{3}=\epsilon_{1} U_{3} U_{1}^{T}, \\
& U_{2} U_{3}=U_{3} U_{2}^{*}, \\
& U_{3} U_{3}^{*}=\epsilon_{2} \mathbf{1} .
\end{aligned}
$$

It follows that the algebra of $\widetilde{U}$ is

$$
\begin{aligned}
\widetilde{U}_{2} \widetilde{U}_{1} & =\widetilde{q} \widetilde{U}_{1} \widetilde{U}_{2}, \\
\widetilde{U}_{3} \widetilde{U}_{1}^{-1} & =\epsilon_{1} \widetilde{U}_{1} \widetilde{U}_{3}, \\
\widetilde{U}_{3} \widetilde{U}_{2} & =\widetilde{U}_{2} \widetilde{U}_{3}, \\
\widetilde{U}_{3} \widetilde{U}_{3} & =\epsilon_{2} \mathbf{1},
\end{aligned}
$$

where $\tilde{q}=e^{\tilde{i} h}$ for $q=e^{i h}$. The case with $q=\epsilon_{1}=\epsilon_{2}=1$ was discussed in the context of heterotic matrix string. Hypermultiplets need to be added for the matrix model to be anomaly free $[12,32]$. It would be interesting to see if other choices lead to any consistent physical theory.

The solution of $X$ on a trivial bundle is

$$
X^{i}=\widetilde{A}^{i}(\widetilde{U}) \widetilde{R}+\widetilde{a}^{i}
$$

where $\widetilde{R}$ and $\widetilde{a}$ 's are derivatives satisfying

$$
\begin{aligned}
\tilde{a}_{i} \widetilde{U}_{j} & =\widetilde{U}_{i}\left(\tilde{a}_{i}+i \delta_{i j}\right), i, j=1,2, \\
\tilde{a}_{i} \widetilde{U}_{3} & =\widetilde{U}_{3} \widetilde{a}_{i}, \\
\widetilde{R} \widetilde{U}_{i} & =\widetilde{U}_{i} \widetilde{R} \\
\widetilde{R} \widetilde{U}_{3} & =-\widetilde{U}_{3} \widetilde{R} .
\end{aligned}
$$




\section{GENERALIZATIONS}

In this section we point out several aspects about matrix theory compactification that are not fully developed in our formulation; more work is needed for better understanding.

\section{A. More general representations}

In previous sections we have assumed that the algebra of $\mathcal{U}$ is realized as a projective regular representation of $\Gamma$ on the $|\Gamma|$ dimensional Hilbert space. This corresponds to the assumption that there is no D-brane located at a fixed point of the quotient space. On the other hand, if there are D-branes located on a fixed point in $\mathbf{R}^{d} / \Gamma$, that is, if the position of the D-brane is invariant under the action of a subgroup $H$ of $\Gamma$, then $U(h)$ should be realized by the unit matrix for all $h \in H$. In Ref. [28] it is nicely demonstrated for the case of $T^{4} / \mathbf{Z}_{2}$ that such representations correspond to the background of D2-branes wrapped on vanishing two-cycles on the orbifold plane. They have also considered direct sums of different representations. In particular the regular representation of $\Gamma$ can be decomposed into a bunch of irreducible representations; thus, one can imagine the meeting of several D-branes at various orbifold planes and merge into a D-particle which can be separated from the orbifold plane [28]. It is expected that their observation can be generalized to other cases as well.

Consider a Hilbert space on which $U(h)=\mathbf{1}$ for all $h$ $\in H$. Then $U(h)$ is central in the algebra of $U$, and so $\alpha(h, g)=\alpha(g, h)=0$. This means that certain choices of the $B$ field background are not consistent with the background of D-branes located at certain singularities.

\section{B. More general quotient conditions}

In the sense that the quotient conditions suggest the more general algebra of $U$ for the matrix compactification, the formulation above also suggests some generalizations of the matrix model.

In the above we have mentioned that taking the quotient of the flat space makes sense only if the map $\Phi$ is a symmetry of the matrix model. If we first compactify the matrix model on a torus and take the limit such that the dual torus becomes an infinite space, we get the gauge field theory on a $d$-dimensional classical space or quantum space, so that the matrix variables $X^{\mu}(\sigma)$ now depend on the coordinates $\sigma$ of the base space. In the decompactification limit the symmetry of Euclidean motions on the base space is restored; so we can impose quotient conditions such as

$$
U(g)^{\dagger} X^{\mu}(\sigma) U(g)=\Phi_{g}^{\mu}\left(X\left(\Lambda_{g}(\sigma)\right)\right),
$$

where $\Lambda_{g}(\sigma)$ is a Euclidean transformation of $\sigma$. This kind of quotient condition is considered in Refs. [36,37] and it is useful in describing a resolution of the singularity in the quotient space.

\section{Noncommutative spacetime to begin with}

Another natural generalization is that since we are allowed to consider noncommutative space in the dual picture, we should also be allowed to consider matrix models in which the entries of the matrix variables are noncommutative from the beginning.

For instance, let $X^{i}=X_{a}^{i} T^{a}$, where $T^{a}$ 's are the Lie algebra generators for $U(n)$ satisfying $\left[T^{a}, T^{b}\right]=i f^{a b c} T^{c}$. We may consider the noncommutative space

$$
\left[X_{a}^{1}, X_{b}^{2}\right]=i h \delta_{a b}
$$

Note that here the original space is already a quantum plane even before we compactify the matrix model on a torus with a background $B$ field. The commutator of $X$ now becomes

$$
\left[X_{1}, X_{2}\right]=\frac{i}{2}\left\{X_{1 a}, X_{2 b}\right\} f^{a b c} T_{c}+i h c_{2} \mathbf{1}
$$

where $c_{2}$ is the second Casimir $\Sigma_{a} T^{a} T^{a}$. Obviously this algebra is invariant under a change of basis of the Lie algebra. This means that the subgroup $\mathbf{Z}_{n}$ of $U(n)$ is preserved and the $n$ D-branes are identical. In addition the algebra is invariant under rotations of $X_{1}$ and $X_{2}$, although the global $\mathrm{SO}(9,1)$ symmetry is broken down to $\mathrm{SO}(2) \times \mathrm{SO}(7,1)$. We can also compactify this model on torus as before since the algebra is invariant under translation. The only modification to our solution (44) is that now the coefficients $A_{g}^{\mu}$ are noncommutative.

Instead of the minimal noncommutativity (150), one may even contemplate on other quantum spaces, for instance those with quantum group symmetries. While these are mathematically natural generalization of the case with $h=0$, its physical interpretation still needs to be identified.

\section{ACKNOWLEDGMENTS}

P.M.H. thanks Professor Chang-Yeong Lee for discussions and people at the Asia Pacific Center for Theoretical Physics for hospitality. His work is supported in part by the National Science Council, Taiwan, R.O.C. Y.S.W. acknowledges the warm hospitality of Laboratoire de Physique Theorique, l'Ecole Normale Superieure in Paris. His work is supported in part by the U.S. National Science Foundation through grant PHY-9601277.
[1] T. Banks, W. Fischler, S.H. Shenker, and L. Susskind, Phys. Rev. D 55, 5112 (1997).

[2] M.R. Douglas and G. Moore, "D-Branes, Quivers and ALE Instantons,', hep-th/9603167.

[3] C.V. Johnson, R.C. Myers, Phys. Rev. D 55, 6382 (1997).
[4] W. Taylor IV, Phys. Lett. B 394, 283 (1997).

[5] O.J. Ganor, S. Ramgoolam, and W. Taylor IV, Nucl. Phys. B492, 191 (1997).

[6] A. Connes, M.R. Douglas, and A. Schwarz, J. High Energy Phys. 02, 003 (1998). 
[7] P.-M. Ho, Y.-Y. Wu, and Y.-S. Wu, Phys. Rev. D 58, 026006 (1998).

[8] P.-M. Ho and Y.-S. Wu, Phys. Rev. D 58, 066003 (1998).

[9] P.-M. Ho and Y.-S. Wu, Phys. Lett. B 398, 251 (1997).

[10] E.G. Gimon and J. Polchinski, Phys. Rev. D 54, 1667 (1996).

[11] M.R. Douglas and C. Hull, J. High Energy Phys. 02, 008 (1998).

[12] T. Banks, N. Seiberg and E. Silverstein, Phys. Lett. B 401, 30 (1997).

[13] A. Fayyazuddin and D.J. Smith, Phys. Lett. B 407, 8 (1997).

[14] N. Kim and S.-J. Rey, Nucl. Phys. B534, 155 (1998).

[15] Y.-K.E. Cheung and M. Krogh, Nucl. Phys. B528, 185 (1998).

[16] A. Connes, Noncommutative Geometry (Academic, New York, 1994).

[17] P.-M. Ho, Phys. Lett. B 434, 41 (1998).

[18] R. Casalbuoni, Phys. Lett. B 431, 41 (1998).

[19] T. Kawano and K. Okuyama, Phys. Lett. B 433, 29 (1998).

[20] F. Ardalan, H. Arfaei, and M.M. Sheikh-Jabbari, "Mixed Branes and M(atrix) Theory on Noncommutative Torus," hep-th/9803067.

[21] B. Morariu and B. Zumino, "Super Yang-Mills on the Noncommutative Torus," hep-th/9807198.

[22] F. Ardalan, H. Arfaei, and M.M. Sheikj-Jabbari, J. High Energy Phys. 02, 016 (1999).
[23] D. Brace, B. Morariu, and B. Zumino, Nucl. Phys. B545, 192 (1999).

[24] M.A. Rieffel, Can. J. Math. 40, 257 (1988).

[25] C. Hofman and E. Verlinde, J. High Energy Phys. 12, 010 (1998).

[26] D. Brace and B. Morariu, J. High Energy Phys. 02, 004 (1999).

[27] J. Polchinski, S. Chaudhuri, and C. Johnson, "Notes On D-Branes,' hep-th/9602052.

[28] S. Ramgoolam and D. Waldram, J. High Energy Phys. 07, 009 (1998).

[29] U.H. Danielsson and G. Ferretti, Int. J. Mod. Phys. A 12, 4581 (1997).

[30] L. Motl, "Quaternions and M(atrix) Theory in Spaces with Boundaries,' hep-th/9612198.

[31] N. Kim and S.-J. Rey, Nucl. Phys. B504, 189 (1997).

[32] T. Banks and L. Motl, J. High Energy Phys. 12, 004 (1997).

[33] S.-J. Rey, Nucl. Phys. B502, 170 (1997).

[34] D.A. Lowe, Phys. Lett. B 403, 243 (1997).

[35] D. Kabat and S.-J. Rey, Nucl. Phys. B508, 535 (1997).

[36] N. Nekrasov and A. Schwarz, Commun. Math. Phys. 198, 689 (1998).

[37] C.I. Lazaroiu, "A Noncommutative-Geometric Interpretation of the Resolution of Equivariant Instanton Moduli Space,' hep-th/9805132. 